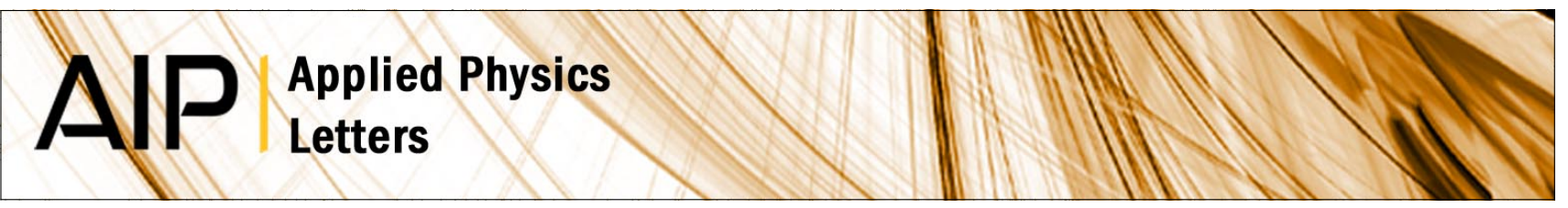

\title{
High-angular-resolution electron energy loss spectroscopy of hexagonal boron nitride
}

R. Arenal, M. Kociak, and N. J. Zaluzec

Citation: Appl. Phys. Lett. 90, 204105 (2007); doi: 10.1063/1.2740185

View online: http://dx.doi.org/10.1063/1.2740185

View Table of Contents: http://apl.aip.org/resource/1/APPLAB/v90/i20

Published by the American Institute of Physics.

\section{Related Articles}

Site-specific mapping of transition metal oxygen coordination in complex oxides

Appl. Phys. Lett. 101, 241910 (2012)

Quantitative phase imaging of electron waves using selected-area diffraction

Appl. Phys. Lett. 101, 234105 (2012)

Removing the effects of elastic and thermal scattering from electron energy-loss spectroscopic data Appl. Phys. Lett. 101, 183112 (2012)

High resolution electron energy loss spectroscopy of clean and hydrogen covered $\mathrm{Si}(001)$ surfaces: First principles calculations

J. Chem. Phys. 137, 094701 (2012)

Nazca Lines by La ordering in La2/3-xLi3xTiO3 ion-conductive perovskite

Appl. Phys. Lett. 101, 073903 (2012)

\section{Additional information on Appl. Phys. Lett.}

Journal Homepage: http://apl.aip.org/

Journal Information: http://apl.aip.org/about/about_the_journal

Top downloads: http://apl.aip.org/features/most_downloaded

Information for Authors: http://apl.aip.org/authors

\section{ADVERTISEMENT}

\section{AIP Applied Physics Letters}

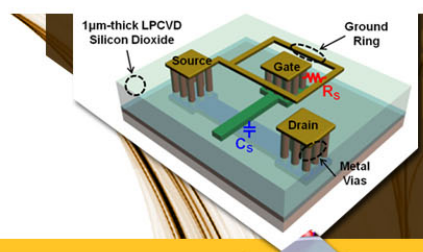

\section{SURFACES AND} INTERFACES

Focusing on physical, chemical, biological structural, optical, magnetic and electrical properties of surfaces and interfaces, and more...

\section{EXPLORE WHAT'S NEW IN APL}

SUBMIT YOUR PAPER NOW!
ENERCY CONVERSION AND STORACE

Focusing on all aspects of static and dynamic energy conversion, energy storage, photovoltaics, solar fuels, batteries, capacitors, thermoelectrics, and more... 


\title{
High-angular-resolution electron energy loss spectroscopy of hexagonal boron nitride
}

\author{
R. Arenal $\left.\right|^{a)}$ \\ Materials Science Division, Argonne National Laboratory, Illinois 60439 \\ M. Kociak \\ Laboratoire de Physique Solides, CNRS/UMR 8502 Université Paris-Sud, 91405 Orsay, France
}

N. J. Zaluzec

Materials Science Division, Argonne National Laboratory, Illinois 60439

(Received 14 February 2007; accepted 23 April 2007; published online 18 May 2007)

\begin{abstract}
High-angular-resolution electron energy loss spectroscopy (EELS) is used to study the anisotropic behavior of the boron and nitrogen $K$ ionization edges in $h$-BN. This work makes significant progress toward improving the anisotropy measurements. The authors show experimentally by EELS the vanishment of the $\pi^{*}$ peak existing in these $K$ edges in agreement with electronic structure calculations and previous soft x-ray absorption spectroscopy measurements. (C) 2007 American Institute of Physics. [DOI: 10.1063/1.2740185]
\end{abstract}

Hexagonal boron nitride $(h$-BN) has a graphitelike structure, with weak van der Waals interactions between the sheets (along [0001] or $c$ axis direction) and strong covalent $s p^{2}$ bonds, partially ionic as the rest of III-V materials, ${ }^{1}$ in the $\{0001\}$ ( $a, b$ axis plane). This ionicity is at the origin of the significant difference between graphite and $h$-BN. Graphite is a semimetal whereas $h$-BN is a large-band-gap semiconductor $^{2}$ and because of this has potential applications in high-temperature, high-power, and high-frequency electronic devices. Recently, large excitonic effects have been observed in $h$-BN (Ref. 3) which make it promising also for applications in optoelectronics as a UV light source. Furthermore, in recent years, the interest of $h$-BN has increased due to the development of techniques to synthesize BN nanotubes (BNNTs). ${ }^{4-6}$

In order to understand the related properties in BNNTs, an improved knowledge of the physical properties of $h$ - BN is necessary. ${ }^{3,7,8,27}$ The study of the near edge fine structure at absorption edges [energy loss near edge structure (ELNES)] using the electron energy loss spectroscopy (EELS) in a transmission electron microscope (TEM) provides a rich source of information on the electronic structure of a material at nanometer scale. ${ }^{9-11}$

In the specific case of a uniaxial anisotropic crystal, such as $h$-BN or graphite, EELS angle-resolved scattering experiments allow researchers to probe this anisotropy in the electronic structure, by selecting a particular scattering vector (or momentum transfer: $\mathbf{q}$ ) in transmission mode. This scattering vector in EELS is equivalent to the polarization vector in $\mathrm{x}$-ray absorption spectroscopy (XAS) experiments. The anisotropic behavior of graphite at the $\mathrm{C}-K$ edge has been studied by EELS either recording EEL spectra at different scattering vectors ${ }^{12,13}$ or using energy-filtered scattering patterns. ${ }^{14}$ These experiments proved the relevance of using EELS to study the anisotropy of the electronic structure of the material. Strikingly, this anisotropy manifests itself by a change in the relative intensity of the different features $\left(\pi^{*}\right.$ and $\sigma^{*}$ peaks) of the $K$ edge. In the case of $\mathrm{BN}$, while a total

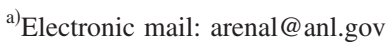

disappearance of the peak $\pi^{*}$ for orientation $\mathbf{q} \perp c$ has been observed in XAS, ${ }^{15,16}$ this has never been the case in EELS in a TEM, even though some experiments have been attempted, ${ }^{12,17,18}$ due to the special experimental conditions required to achieve this goal. ${ }^{19,20}$ This electronic anisotropy has also been studied both theoretically and modeled to compare with experiments. ${ }^{21}$

In this work, we present the angle-resolved EELS measurements on highly pure $h$-BN microcrystals using high angle-resolved electron channeling spectroscopy (HARECES) technique. ${ }^{22}$ In this study, we show for an EELS experiment in a TEM the complete vanishment of the $\pi^{*}$ peak for the boron and nitrogen $K$ edges of $h$-BN.

We performed these studies in a Tecnai F-20 microscope operating at $200 \mathrm{kV}$. The energy resolution was close to $1.5 \mathrm{eV}$ (full width at half maximum of the "elastic" peak) and the collection semiangle $(\beta)$ of $0.11 \mathrm{mrad}$. We used a high coherent and nearly parallel probe $(\alpha<0.05 \mathrm{mrad})$. The diameter of this probe was less than $1 \mu \mathrm{m}$ and it is positioned on a single $\{0001\}$ oriented grain of $h$-BN, as we can see in the selected area diffraction pattern shown in Fig. 1(a). The angular resolution was better than 0.05 mrad. Customized control computer software was employed to select the scattering direction for measurements. ${ }^{22}$ All the spectra were corrected for dark current and detector gain variations and the background was subtracted using the standard power law


FIG. 1. (a) $\{0001\}$ selected area electron diffraction patterns of a single crystal of $h$-BN. Marker: scattering angle, and line: angular scan direction for the data shown in (b). (b) Surface plot of the orientation dependence (symmetric orientation of the $\langle 11 \overline{2} 0\rangle$ systematic row) of the backgroundcorrected boron and nitrogen $K$ edges. 


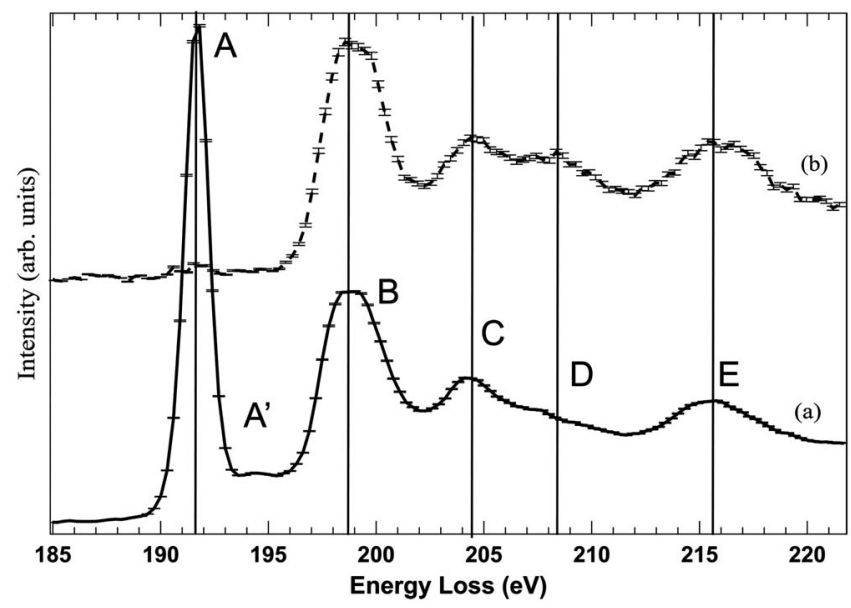

FIG. 2. Boron $K$ ionization edge for two scattering angles of (a) $0 \mathrm{mrad}$ and (b) $1.49 \mathrm{mrad}$ in the $\langle 11 \overline{2} 0\rangle$ direction, respectively. Standard error bars are shown on the data.

methodology. ${ }^{9}$ The relative specimen thicknesses $(t / \lambda)$ was 0.4 , measured with a scattering angle of $0.3 \mathrm{mrad}$ in the $\langle 11 \overline{2} 0\rangle$ direction.

The samples used in this work were highly pure micropowders of $h$-BN (Goodfellow) that we sonicated in ethanol and deposited onto a holey amorphous carbon film supported by a copper TEM grid. Typical EELS acquisition times were several hours (from 1 to $5 \mathrm{~h}$ ), in order to mitigate hydrocarbon contamination during the acquisition, the specimen and grid were cleaned before the analysis using an argon plasma cleaner system (South Bay Tech. PC2000) for 20 min. $^{23}$ During all the experiments no damage was observed nor was there any contamination, as we can see in Fig. 1(b), where no carbon is detected (C- $K$ edge at $\sim 284 \mathrm{eV}$ ).

As we mentioned earlier, $h$-BN is a layered crystal where the in-plane atoms are bonded through localized covalent $s p^{2}$ hybridization, while the out-of-plane layers are bonded by delocalized weak $p$ orbitals. The fine structure in B- $K$ and $\mathrm{N}-K$ edges depends strongly on the orientation of the scattering angle with respect to the anisotropic axis $(c$, perpendicular to the $\mathrm{BN}$ planes). ${ }^{12}$ We probe this orientation dependence using a set of different scattering angles for the $\{0001\}$ oriented microcrystallite. We also note that for the small scattering angles considered in these measurements $(\theta \leqslant 5 \mathrm{mrad})$ the dipole selection rules are valid. ${ }^{12}$

ELNES features associated with the B- $K$ and $\mathrm{N}-K$ ionization edges in $h$-BN are displayed in Figs. 2 and 3 for two different scattering angles, one at the $\gamma$ point (0001) and the second one along the direction $\langle 11 \overline{2} 0\rangle$ [marked by the arrow in Fig. 1(a)]. The positions of the main features [labeled (A-E) for B- $K$ edge and (I-VI) for $\mathrm{N}-K$ edge, respectively] observed in Figs. 2 and 3 are listed in Table I. These values correspond very well with those from the literature (XAS). ${ }^{24}$ Both edges, as well as their angular variation, look qualitatively similar. Their resemblance is a consequence of the strong covalent bonding in $\mathrm{BN}^{25}$ For a given edge, the difference between the two spectra ((a) and (b)) is striking. While the first peak (A or I) totally disappears at large scattering angle, the structures at higher energy change only slightly with scattering angle. A quantitative description of this effect is shown in Fig. 4(a) for the peaks A and B of the

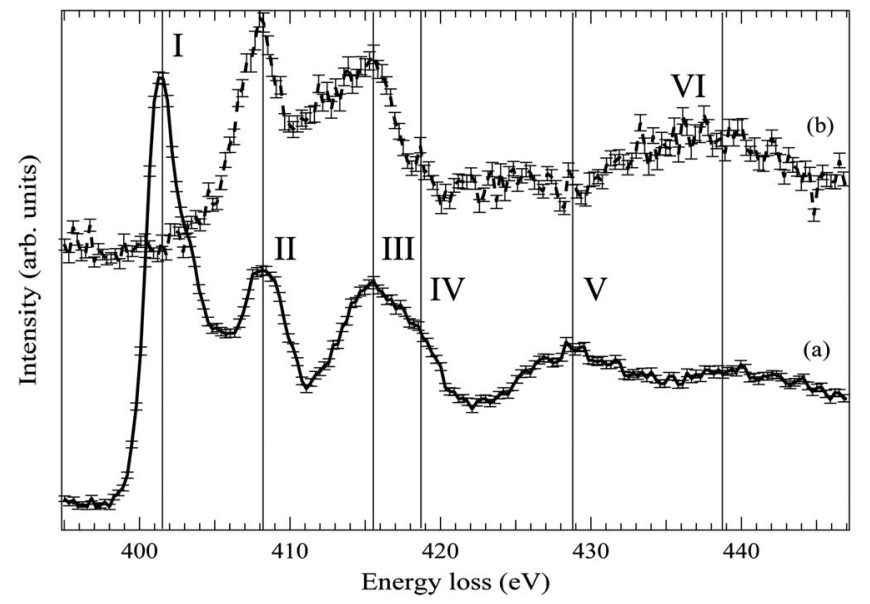

FIG. 3. Nitrogen $K$ ionization edge for two scattering angles of (a) $0 \mathrm{mrad}$ and (b) $1.49 \mathrm{mrad}$ in the $\langle 11 \overline{2} 0\rangle$ direction, respectively. Standard error bars are shown on the data.

B- $K$ edge and in Fig. 4(b) for the peaks I and II of the N-K edge. At large scattering angle, peak A (I) decreases much faster than peak B (II), as expected from the former analysis, while at small scattering angle, energy peak B (II) presents two maxima as a function of the scattering angle.

We now turn to the interpretation of the different peaks. The prominent, narrow, and intense peaks $\mathrm{A}$ and $\mathrm{I}$ in Figs. 2(a) and 3(a) correspond to the transitions of the B and $\mathrm{N}$, respectively, with $1 s$ electron to the empty $\pi^{*}$ antibonding orbitals, which are associated with $s p^{2}$ hybridization and planar bonding. Although it is important to note that in the case of $\mathrm{N}-K$ edge the nature of the first peak (I) is different from the core exciton in the boron case (A peak). The resonances of the B and II peaks correspond to the transitions of the boron and nitrogen, respectively, with $1 s$ electron to the $\sigma^{*}$ orbital which is double degenerate $\left(2 s p_{x, y}\right)$. Experimental conditions did not permit the observation of this degeneracy. ${ }^{26}$ Concerning the C-E (III-V) peaks, they are associated with the $1 s$ electron transitions to the $\sigma^{*}$ orbitals. The peak VI in the N-K ionization edge is part of the extended energy loss fine structure due to backscattering from the nearest neighbors. ${ }^{9}$

In our measurements we observe that for large scattering angle A and I, peaks become negligibly small compared to B and II peaks. This is clearly an anisotropic effect: we have measured (data not shown) that, as expected from theory, ${ }^{18}$ the ratio between $\pi^{*}$ and $\sigma^{*}$ peaks is proportional to $\left(\theta / \theta_{E}\right)^{2}$, where $\theta_{E}$ is the characteristic inelastic angle [at $200 \mathrm{kV}$, $\theta_{E}(\mathrm{~B}-K$ edge $)=0.53 \mathrm{mrad}$ and $\theta_{E}(\mathrm{~N}-K$ edge $\left.)=1.14 \mathrm{mrad}\right]$. This is not surprising, since $\left(\theta / \theta_{E}\right)$ is the ratio of the components of the scattering angle oriented, respectively, along

TABLE I. Energy positions of the different peaks present in the B- $K$ and $\mathrm{N}-K$ edges.

\begin{tabular}{cccc}
\hline \hline Feature & Position (eV) & Feature & Position $(\mathrm{eV})$ \\
\hline A & 191.9 & I & 401.6 \\
A $^{\prime}$ & 194.3 & II & 408.1 \\
B & 198.1 & III & 415.4 \\
C & 204.0 & IV & 418.8 \\
D & 207.4 & V & 428.6 \\
E & 215.4 & VI & 438.5 \\
\hline \hline
\end{tabular}



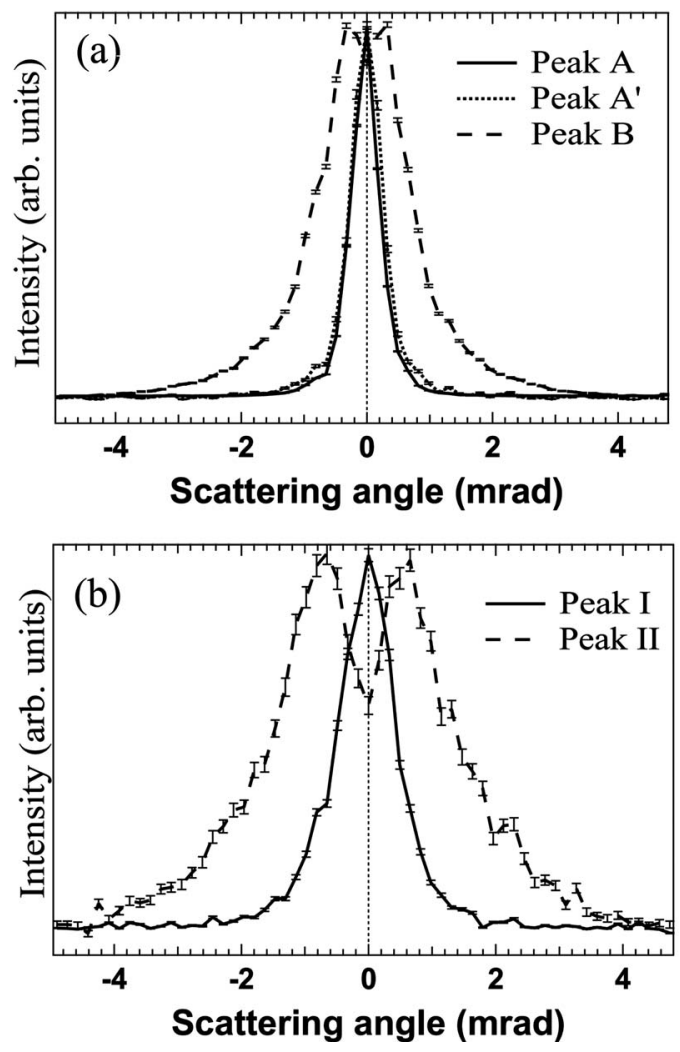

FIG. 4. Orientation dependence of (a) A, $\mathrm{A}^{\prime}$, and $\mathrm{B}$ peaks of the $\mathrm{B}-K$ edge and (b) I and II peaks of the N-K edge. Standard error bars are shown on the data.

the $\pi$ and $\sigma$ orbitals. It might be tempting to use the same argument at zero scattering angle. However, at small scattering angle, the finite size of the collection aperture $(\beta)$ of the spectrometer average out the anisotropic effect and $\sigma^{*}$ peak does not drop to zero. ${ }^{14}$ Nevertheless, a clear indication of the anisotropic character is given by the Mexican hat shape of the $\sigma^{*}$ peak with respect to the scattering angle (Fig. 4), as previously predicted ${ }^{18}$ and measured for graphite ${ }^{12,14}$ and the B- $K$ edge of $h$-BN. ${ }^{12}$

Another observation is the peak $\mathrm{A}^{\prime}$ which is only present in the B-K edge [Fig. 2(a)] and is clearly visible for this particular orientation (q parallel to $c$ axis). This peak appears as a shoulder for A peak and has the same behavior as this A peak, see Fig. 4(a). Thus, we conclude that this $\mathrm{A}^{\prime}$ peak is associated with a transition to $p_{z}$ final states and could correspond to the beginning of the $\pi^{*}$ band which is shifted to low energy due to the effect of the core-hole interaction. Nevertheless, this aspect needs further theoretical investigation. We also note the behavior of the $\mathrm{V}$ peak in the $\mathrm{N}-K$ edge which is only visible for the orientation $\mathbf{q}$ parallel to the $c$ axis, Fig. 3(a).

In conclusion, we have acquired angle-resolved EELS on a single crystal of $h$-BN. These results are in complete agreement with the calculations and significantly improve on the older work. Furthermore, this study demonstrated that
HARECES is a powerful technique to perform momentum resolved EELS in a TEM and allows one to investigate the symmetry of electronic excitations in materials. We showed for an EELS experiment in a TEM that the $\pi^{*}$ resonance of the B- $K$ and $\mathrm{N}-K$ ionization edges drops to zero. These results will be very useful to enable a better understanding of the electronic band structure of the BNNTs where the local anisotropy effects are stronger.

This work was supported by the U.S. Department of Energy, Office of Science, under Contract No. DE-AC0206CH11357 and was carried out in the Electron Microscopy Center at Argonne National Laboratory. The authors would like to thank O. Stephan for many helpful discussions.

${ }^{1}$ P. Atkins and J. de Paula, Physical Chemistry, 7th ed. (Oxford University Press, Oxford, 2002), 428.

${ }^{2}$ A. Rubio, J. L. Corkill, and M. L. Cohen, Phys. Rev. B 49, 5081 (1994).

${ }^{3}$ L. Wirtz, A. Marini, and A. Rubio, Phys. Rev. Lett. 96, 126104 (2006).

${ }^{4}$ R. S. Lee, J. Gavillet, M. Lamy de la Chapelle, A. Loiseau, J.-L. Cochon, D. Pigache, J. Thibault, and F. Willaime, Phys. Rev. B 64, 121405(R) (2001).

${ }^{5}$ R. Arenal, Ph.D. thesis, Université Paris XI-Orsay, 2005.

${ }^{6}$ R. Arenal, M. Kociak, A. Loiseau, and D. J. Miller, Appl. Phys. Lett. 89, 073104 (2006).

${ }^{7}$ R. Arenal, A. C. Ferrari, S. Reich, L. Wirtz, J.-Y. Mevellec, S. Lefrant, A. Rubio, and A. Loiseau, Nano Lett. 6, 1812 (2006).

${ }^{8}$ R. Arenal, O. Stephan, M. Kociak, D. Taverna, A. Loiseau, and C. Colliex, Phys. Rev. Lett. 95, 127601 (2005).

${ }^{9}$ R. F. Egerton, Electron Energy-Loss Spectroscopy in the Electron Microscope, 2nd ed. (Plenum, New York, 1996), 338-350.

${ }^{10}$ R. Arenal, O. Stephan, M. Kociak, D. Taverna, C. Colliex, A. Rubio, and A. Loiseau, AIP Conf. Proc. 723, 293 (2004).

${ }^{11}$ R. Arenal, O. Stephan, A. Loiseau, and C. Colliex (unpublished).

${ }^{12}$ R. D. Leapman, P. L. Fejes, and J. Silcox, Phys. Rev. B 28, 2361 (1983).

${ }^{13}$ N. J. Zaluzec, M. G. Blackford, K. L. Smith, and M. Colella, Microsc. Microanal. 11, 718 (2005).

${ }^{14}$ G. Radtke, G. A. Botton, and J. Verbeeck, Ultramicroscopy 106, 1082 (2006).

${ }^{15}$ N. Watanabe, H. Hayashi, Y. Udagawa, K. Takeshita, and H. Kawata, Appl. Phys. Lett. 69, 1370 (1996).

${ }^{16}$ J. Moscovici, G. Loupias, Ph. Parent, and G. Tourillon, J. Phys. Chem. Solids 57, 1159 (1996).

${ }^{17}$ H. K. Schmid, Microsc. Microanal. Microstruct. 6, 99 (1995).

${ }^{18}$ C. Souche, B. Jouffrey, G. Hug, and M. Nelhiebel, Micron 29, 419 (1998).

${ }^{19}$ G. A. Botton, C. B. Boothroyd, and W. M. Stobbs, Ultramicroscopy 59, 93 (1995).

${ }^{20}$ V. J. Keast, A. J. Scott, R. Brydson, D. B. Williams, and J. Bruley, J. Microsc. 203, 135 (2001).

${ }^{21}$ I. Tanaka, H. Araki, M. Yoshiya, T. Mizoguchi, K. Ogasawara, and H. Adachi, Phys. Rev. B 60, 4944 (1999).

${ }^{22}$ N. J. Zaluzec, Microsc. Microanal. 6, 938 (2000).

${ }^{23}$ N. J. Zaluzec, B. K. Kernstel, and D. Henriks, Microsc. Microanal. 3, 983 (1997).

${ }^{24}$ R. Franke, S. Bender, J. Hormes, A. A. Pavlychev, and N. G. Fominych, Chem. Phys. 216, 243 (1997).

${ }^{25}$ L. A. J. Garvie, H. Hubert, P. Rez, P. F. McMillan, and P. R. Buseck, J. Alloys Compd. 290, 34 (1998).

${ }^{26}$ In order to acquire simultaneously B- $K$ and N- $K$ edges, we used an energy dispersion of $0.3 \mathrm{eV}$ and because of this degeneracy is not clearly visible in our spectra.

${ }^{27}$ J. Serrano, A. Bosak, R. Arenal, M. Krisch, K. Watanabe, T. Taniguchi, and H. Kanda, Phys. Rev. Lett. 98, 095503 (2007). 